\title{
Wilms' tumor gene 1 is an independent prognostic factor for pediatric acute myeloid leukemia following allogeneic hematopoietic stem cell transplantation
}

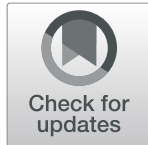

Dao-Xing Deng ${ }^{1 \dagger}$, Juan-Juan Wen ${ }^{1,2+}$, Yi-Fei Cheng ${ }^{1}$, Xiao-Hui Zhang ${ }^{1}$, Lan-Ping X ${ }^{1}$, Yu Wang ${ }^{1}$, Chen-Hua Yan ${ }^{1}$, Yu-Hong Chen ${ }^{1}$, Huan Chen ${ }^{1}$, Wei Han ${ }^{1}$, Feng-Rong Wang ${ }^{1}$, Jing-Zhi Wang ${ }^{1}$, Ya-Zhen Qin ${ }^{1}$, Kai-Yan Liu', Xiao-Jun Huang ${ }^{1,3,4}$, Xiao-Su Zhao ${ }^{1,4^{*}}$ and Xiao-Dong Mo ${ }^{1 *}$

\footnotetext{
Abstract

Background: Sequential monitoring of Wilms' tumor gene 1 (WT1) after allogeneic hematopoietic stem cell transplantation (allo-HSCT) could predict relapse in adult acute myeloid leukemia (AML). However, the prognostic role of WT1 in pediatric AML after allo-HSCT is unclear. Thus, we determined to see whether sequential monitoring of WT1 after allo-HSCT could predict relapse in AML children.

Methods: Pediatric AML patients receiving allo-HSCT from January 21, 2012 to December 20, 2018 at the Peking University Institute of Hematology were included in this study. WT1 expression level was determined by TaqManbased reverse transcription-polymerase chain reaction. WT1 sequential monitoring was performed 1, 2, 3, 4.5, 6, 9, and 12 months post-transplantation and at 6-month intervals thereafter. The primary end point was relapse. The secondary end points included disease-free survival (DFS), overall survival (OS), and non-relapse mortality (NRM). Kaplan-Meier analysis was used for DFS and OS estimates, while competing risk analysis was used for estimating relapse and NRM.

(Continued on next page)
}

\footnotetext{
*Correspondence: zhao.xiaosu@outlook.com; mxd453@163.com

${ }^{\dagger}$ Dao-Xing Deng and Juan-Juan Wen contributed equally to this work.

${ }^{1}$ Peking University People's Hospital, Peking University Institute of Hematology, National Clinical Research Center for Hematologic Disease,

Research Unit of Key Technique for Diagnosis and Treatments of Hematologic Malignancies, Chinese Academy of Medical Sciences, 2019RU029, Beijing Key Laboratory of Hematopoietic Stem Cell Transplantation, Beijing, China

Full list of author information is available at the end of the article
}

(c) The Author(s). 2021 Open Access This article is licensed under a Creative Commons Attribution 4.0 International License, which permits use, sharing, adaptation, distribution and reproduction in any medium or format, as long as you give appropriate credit to the original author(s) and the source, provide a link to the Creative Commons licence, and indicate if changes were made. The images or other third party material in this article are included in the article's Creative Commons licence, unless indicated otherwise in a credit line to the material. If material is not included in the article's Creative Commons licence and your intended use is not permitted by statutory regulation or exceeds the permitted use, you will need to obtain permission directly from the copyright holder. To view a copy of this licence, visit http://creativecommons.org/licenses/by/4.0/ The Creative Commons Public Domain Dedication waiver (http://creativecommons.org/publicdomain/zero/1.0/) applies to the data made available in this article, unless otherwise stated in a credit line to the data. 
(Continued from previous page)

Results: Of the 151 consecutive patients included, the median age was 10 years (range, 1-17). The optimal cutoff value of WT1 within 1 year after allo-HSCT to predict relapse was 0.8\% (80 WT1 copies/10 4 ABL copies), with a sensitivity of $60 \%$ and specificity of $79 \%$. Compared with WT1 expression $<0.8 \%$, WT1 expression $\geq 0.8 \%$ indicated significantly higher 5 -year cumulative incidence of relapse (CIR, 35.1\% vs. 11.3\%; $P=0.001)$, lower 5-year disease-free survival (DFS, 60.4\% vs. 80.8\%; $P=0.009$ ), and lower 5 -year overall survival (OS, 64.9\% vs. 81.6\%; $P=0.038$ ) rates. Multivariate analyses showed that WT1 was an independent risk factor for relapse (HR 2.89; $95 \%$ confidence interval $(C I), 1.25-6.71 ; P=0.014)$. Both the CIR (5-year CIR: 8.3\% vs. 11.3\%; $P=0.513$ ) and DFS (5-year DFS: $91.7 \%$ vs. 80.8\%; $P=0.208)$ were comparable between patients achieving minimal residual disease (MRD) negativity after preemptive interferon-a (IFN-a) treatment and those without MRD after allo-HSCT, which were better than those of MRDpositive patients without preemptive therapies.

Conclusions: Sequential monitoring of WT1 could predict relapse in pediatric AML after allo-HSCT. WT1-directed immunotherapy may have the potential to prevent relapse and improve survival.

Keywords: Pediatric, Acute myeloid leukemia, Allogeneic hematopoietic stem cell transplantation, Wilms' tumor gene 1, Relapse

\section{Background}

Allogeneic hematopoietic stem cell transplantation (alloHSCT) is one of the most critical therapies for pediatric acute myeloid leukemia (AML) patients, and many of them can achieve long-term disease-free survival (DFS) $[1,2]$. However, relapse remains the most important cause of transplantation failure [3]. Therefore, preventing relapse is important to improve the outcomes of pediatric AML patients receiving allo-HSCT.

Minimal residual disease (MRD) monitoring is the most important method to recognize the early presentations of relapse, which could help to prevent posttransplant relapse [4]. MRD monitoring methods include multiparameter flow cytometry (MFC) and polymerase chain reaction (PCR) [5]. MFC monitoring is critical for AML patients, but AML carries multiple immunophenotypic clones and frequently changes the antigen profile after therapies, which restricts the application of MFCMRD in AML patients [6]. PCR is another important method for MRD monitoring, particularly for those with leukemic fusion genes [7]. Currently, $P M L-R A R \alpha$, RUNX1-RUNX1T1,CBF $\beta-M Y H 11$, and NPM1 mutation are recommended for use as molecular markers for MRD monitoring [5]; however, only $30-40 \%$ of pediatric AML patients have these molecular markers [8]. Therefore, for patients without mature molecular MRD markers, ways to monitor MRD should be further identified.

Wilms' tumor gene 1 (WT1) is highly expressed in most acute leukemia patients and can be quantitatively detected, enabling it to be a potential MRD marker for pediatric AML without special molecular markers [7]. Some studies have shown that WT1 could predict relapse in AML children receiving chemotherapy $[9,10]$. In addition, some studies showed that WT1 could predict post-transplant relapse of AML [11-14], but the samples of children enrolled in these studies were small. Meanwhile, some authors observed that the expression of WT1 was different between children and adults [15]. In a word, for relapse prediction of pediatric AML after allo-HSCT, data of WT1 expression was in urgent need. To the best of our knowledge, only one study explored the role of WT1 expression in relapse prediction in pediatric AML after allo-HSCT [16]. This study found that WT1 failed to predict relapse in pediatric AML after allo-HSCT. However, this study only involved 2 time points $(+42 \mathrm{~d}$ and $+100 \mathrm{~d})$ of WT1 monitoring. In addition, previous studies showed that sequential monitoring of WT1 after allo-HSCT could predict relapse in adult AML patients $[11,12]$. Therefore, because of insufficient time points, the conclusion of meaningless WT1 monitoring in pediatric AML after allo-HSCT could not be made. To sum up, WT1 sequential monitoring was critical to predict relapse in AML patients after alloHSCT, but whether sequential monitoring of WT1 could predict relapse of AML children after allo-HSCT was unclear.

Therefore, we aimed to identify the prognostic value of WT1 sequential monitoring in pediatric AML patients after allo-HSCT. In particular, we wanted to identify the cut-off value of WT1 for predicting relapse after alloHSCT in AML children.

\section{Methods \\ Patients}

From January 21, 2012 to December 20, 2018, 151 consecutive pediatric AML patients receiving allo-HSCT at the Peking University Institute of Hematology (PUIH) were enrolled in this study with the following criteria: (1) < 18 years old; (2) diagnosed with AML without PML-RAR $\alpha, \quad R U N X 1-R U N X 1 T 1, \quad C B F \beta-M Y H 11$, or NPM1 mutation; and (3) monitored WT1 expression 
regularly after allo-HSCT. This study followed the principles of the Helsinki Declaration and was approved by the ethical committee of Peking University People's Hospital.

\section{Transplantation regimens}

The preconditioning regimens consisted of cytarabine (Ara-C), busulfan, cyclophosphamide, and simustine. Rabbit anti-thymocyte globulin was administered to the HLA-unrelated donor (URD), HLA-haploidentical related donor (haplo-RD), and umbilical cord blood (UCB) donor groups (eMethods in the Supplement) [17-19]. Patients received cyclosporine A (CsA), mycophenolate mofetil (MMF), and short-term methotrexate (MTX) as GVHD prophylaxis. UCB transplantation recipients received methylprednisolone (MP) instead of MTX [19, 20]. Donor selection, HLA typing, and stem cell harvesting have been described in detail elsewhere [21].

\section{MRD monitoring and definition}

MRD monitoring was based on leukemia-associated aberrant immune phenotypes (LAIPs) detected by MFC [22] and WT1 expression levels determined through TaqMan-based RQ-PCR technology (eMethods in the Supplement) [11]. MFC positivity was defined as $>0.1 \%$ of cells with a LAIP in post-transplantation bone marrow (BM) samples according to the ELN criteria [5]. The transcript level was calculated as WT1 transcript copies/ ABL copies in percentage. According to our experiment method, the transcript level of WT1 could also be calculated as WT1 copies $/ 10^{4}$ ABL copies. Routine MRD monitoring was performed $1,2,3,4.5,6,9$, and 12 months post-transplantation and at 6-month intervals thereafter. MRD positivity was defined as MFC positivity or WT1 positivity. WT1 positivity alone (WT1+ alone) was defined as WT1 positivity without MFC positivity in BM samples. Combined MRD positivity (MRDco+) was defined as both MFC positivity and WT1 positivity in a BM sample. Relapse was defined as the recurrence of $>$ $5 \%$ BM blasts, the reappearance of blasts in the blood or the development of extramedullary disease. We defined WT1 positivity except relapse. The data of WT1 expression at the time of relapse would not be considered.

\section{Preemptive intervention}

A part of patients included in the study ever entered a prospective clinical study exploring the efficacy of interferon- $\alpha$ (IFN- $\alpha$ ) treatment from 2012 to 2014, and results of the study had been published [23]. Since then, IFN- $\alpha$ treatment was a routine for patients with MRD positivity at our center. Moreover, IFN- $\alpha$ treatment was also one of the preemptive interventions recommended by the consensus about treatment and prevention of leukemia relapse after allo-HSCT in China [3].
Chemotherapy plus donor lymphocyte infusion (ChemoDLI) was a routine for patients with MRD positivity at our center since 2011 [24]. The protocol of IFN- $\alpha$ treatment $[23,25-28]$ and Chemo-DLI $[24,29,30]$ was summarized in the eMethods in the Supplement. Informed consent to preemptive intervention was gotten from all patients' guardian. In addition, 26 patients with WT1 positivity did not receive preemptive intervention. Among them, two patients had active aGVHD, five patients had active cGVHD, two patients had active infections, and 17 patients refused to receive preemptive intervention since the prognostic role of WT1 expression was uncertain in pediatric AML after allo-HSCT before this study.

\section{Statistical methods}

The last follow-up date was 31 December 2019. The primary end point was relapse. The secondary end points included DFS, overall survival (OS), and non-relapse mortality (NRM). Relapse, DFS, OS and NRM were calculated from the date of transplantation. Relapse was defined as the recurrence of $>5 \%$ BM blasts, the reappearance of blasts in the blood or the development of extramedullary disease. Deaths were the events for OS. The events for DFS included relapse and death of any cause. Kaplan-Meier analysis was used for DFS and OS estimates, with the log-rank test used for comparisons between groups. Competing risk analysis was used for estimating relapse and NRM, and the Gray's test was applied for comparisons between subgroups. NRM was the competing event for relapse and vice versa. Cox's proportional hazards model was used for multivariable analyses. $P<0.05$ was considered statistically significant. Analyses were performed using SPSS 20.0 (Mathsoft, Seattle, WA), GraphPad Prism 6 (GraphPad Software Inc., La Jolla, CA) and R software (http://cran.R-project.org).

\section{Results \\ Patient characteristics}

A total of 151 consecutive pediatric AML patients were enrolled (Table 1). The median age was 10 years (range, 1-17) and the median follow-up time was 798 days (range, 55-2901) after allo-HSCT. Thirty-one patients experienced relapse, and nine patients suffered TRM. In total, we collected 1242 BM samples from these patients after allo-HSCT.

\section{The expression of WT1 in patients without relapse after allo-HSCT}

A total of 81 patients did not receive any intervention and showed persistent complete remission (CR), and 501 $\mathrm{BM}$ samples were collected from these patients within 1 year after allo-HSCT. The median WT1 expression levels at $+1,+2,+3,+4.5,+6,+9$, and 12 months were 
Table 1 Patient characteristics $(n=151)$

\begin{tabular}{|c|c|}
\hline Characteristics & $N=151$ \\
\hline Gender (male/female) & $89 / 62$ \\
\hline Median age (years, range) & $10(1-17)$ \\
\hline \multicolumn{2}{|l|}{ FAB type } \\
\hline MO & 5 \\
\hline M1 & 4 \\
\hline M2 & 43 \\
\hline M4 & 17 \\
\hline M5 & 57 \\
\hline M6 & 11 \\
\hline M7 & 10 \\
\hline MDS-AML & 3 \\
\hline Therapy-related AML & 1 \\
\hline \multicolumn{2}{|l|}{ Cytogenetics and molecular abnormalities } \\
\hline Intermediate & 94 \\
\hline Unfavorable & 57 \\
\hline \multicolumn{2}{|l|}{ Disease status before allo-HSCT } \\
\hline CR1 & 132 \\
\hline CR2 & 15 \\
\hline NR & 4 \\
\hline \multicolumn{2}{|l|}{ Donor type } \\
\hline ISD & 13 \\
\hline URD & 5 \\
\hline Haplo-RD & 129 \\
\hline UCB & 4 \\
\hline Median MNC (×10^8/kg, range) & $8.57(0.59-16.66)$ \\
\hline Median CD34 (×10^6/kg, range) & $2.85(0.17-10.95)$ \\
\hline NE engraftment $(n, \%)$ & $151(100.0 \%)$ \\
\hline Median time from HSCT to NE engraftment (days, range) & $12(10-23)$ \\
\hline PLT engraftment $(n, \%)$ & $146(96.7 \%)$ \\
\hline Median time from HSCT to PLT engraftment (days, range) & $15.5(7-128)$ \\
\hline
\end{tabular}

CR Complete remission, NR No remission, ISD HLA-identical sibling donor, URD HLA-unrelated donor, Haplo-RD HLA-haploidentical related donor, UCB Umbilical cord blood, MNC Mononuclear cell, NE Neutrophil, PLT Platelet

$0.120 \%$ (0.000-8.600\%), $0.170 \%$ (0.000-1.000\%), $0.160 \%$ (0.016-2.500\%), 0.290\% (0.014-2.000\%), 0.250\% (0.059$6.200 \%), 0.300 \%(0.035-1.400 \%)$, and $0.290 \%$ (0.016$1.700 \%)$, respectively (Fig. 1).

\section{The cutoff value of WT1 for relapse prediction}

The highest expression level of WT1 within 1 year after allo-HSCT was the highest one in seven time points (1, $2,3,4.5,6,9$, and 12 months post-transplantation). If a patient relapsed within 1 year after allo-HSCT, only the values of WT1 expression before relapse would be used for picking out the highest expression level of WT1. We carried out ROC analysis to explore the association between relapse and the highest expression level of WT1 within 1 year after allo-HSCT in 96 patients who did not receive any preemptive interventions. The area under the ROC curve was 0.697 (95\% confidence interval (CI) $=0.541-0.854, P=0.016$; eFigure 1 in the Supplement). The optimal cutoff value of WT1 within 1 year after HSCT to predict relapse was $0.8 \%$ (80 WT1 copies $/ 10^{4}$ ABL copies), with a sensitivity of $60 \%$ and specificity of 79\% (eTable 1 in the Supplement).

\section{The association between WT1 and MFC positivity within 1 year after allo-HSCT}

We defined WT1 positivity as WT1 $\geq 0.8 \%$. Spearman correlation analysis showed that WT1 positivity was significantly associated with MFC positivity (Spearman's 


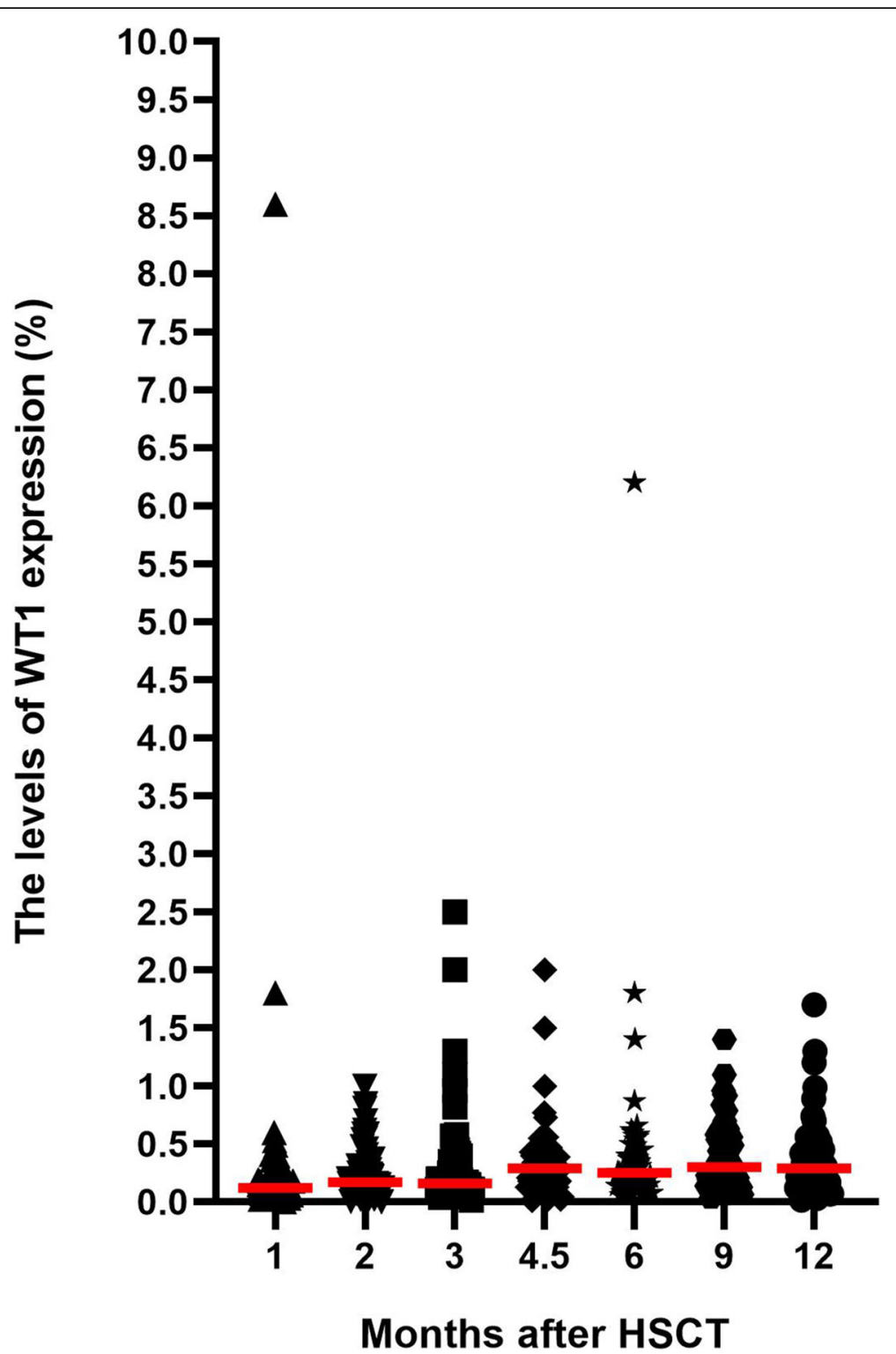

Fig. 1 WT1 expression at different points after allo-HSCT in patients maintaining CR without interventions $(n=81)$. Horizontal bars show the median values of WT1 at each time point

correlation coefficient: $0.344, P<0.001$ ) within 1 year after allo-HSCT. Among the 67 patients with WT1 positivity, 9 showed WT1 and MFC positivity simultaneously. Four patients showed WT1 positivity 28, 42, 148, and 244 (median: 95) days prior to MFC positivity, respectively.

WT1 positivity within 1 year after allo-HSCT predicted poor outcomes

Among the patients without preemptive interventions after allo-HSCT $(n=96)$, patients with WT1 positivity within 1 year after allo-HSCT had a significantly higher 2-year cumulative incidence of relapse (CIR, $36.2 \%$ vs. $9.2 \% ; P=0.002$ ), a significantly lower 2 -year probability of DFS $(59.9 \%$ vs. $81.4 \% ; P=0.018)$, and a trend of a lower 2-year probability of OS $(68.2 \%$ vs. $84.5 \% ; P=0.068)$ than those with WT1 negativity (Fig. 2). The 2-year CIR of patients with WT1+ alone (28.4\% vs. 9.2\%; $P=0.032)$ and MRDco+ $(57.1 \%$ vs. 9.2\%; $P<0.001$ ) were both significantly higher than that of those without MRD. In addition, the 2-year CIR was comparable between the WT1+ alone and MRDco+ groups (28.4\% vs. 57.1\%; $P=0.168$ ) (Fig. 3a). Multivariate analyses showed that WT1 positivity after allo-HSCT was an independent risk factor for relapse (HR 6.15; 95\% confidence interval (CI), 1.62-23.33; $P=0.008)$ and DFS (HR 2.97; 95\% CI, 1.05-8.46; $P=0.041$ )

(Table 2). 

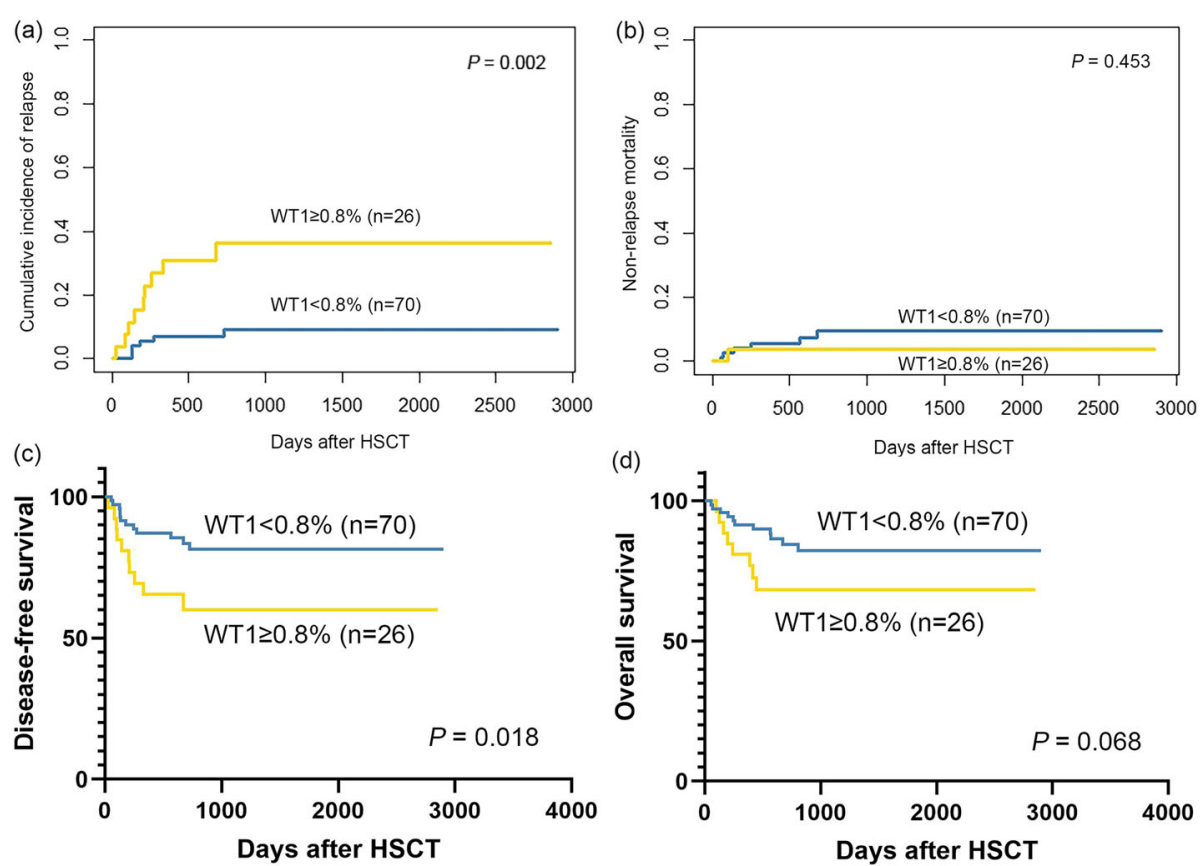

Fig. 2 The outcomes of patients without preemptive interventions according to WT1 after allo-HSCT $(n=96)$ : a relapse, b non-relapse mortality, c disease-free survival, and $\mathbf{d}$ overall survival

In total, 22 patients with WT1 positivity within 1 year after allo-HSCT suffered relapse. The median interval from WT1 positivity to relapse was 105 (13714) days. In addition, seven patients with MFC and WT1 positivity (MRDco+) after allo-HSCT suffered relapse. The median interval from MRDco+ to relapse was $58(13-550)$ days. In the total cohort $(n=151)$, patients with WT1 positivity within 1 year after alloHSCT had a significantly higher 5 -year CIR $(35.1 \%$ vs. $11.3 \% ; \quad P=0.001)$, a lower 5 -year DFS $(60.4 \%$ vs. $80.8 \% ; P=0.009)$, and a lower 5 -year OS $(64.9 \%$ vs.
81.6\%; $P=0.038)$ than those with WT1 negativity (eFigure 2 in the Supplement). The 5-year CIR of patients with WT1+ alone $(29.8 \%$ vs. $11.3 \% ; P=0.013)$ and MRDco+ $(55.8 \%$ vs. $11.3 \% ; P<0.001)$ were both significantly higher than that of those without MRD. In addition, the 5-year CIR of patients with WT1+ alone tended to be lower than that of patients with MRDco+ $(29.8 \%$ vs. $55.8 \% ; P=0.060)$ (Fig. $3 b)$. Multivariate analyses showed that WT1 positivity after alloHSCT was an independent risk factor for relapse (HR 2.89; 95\% CI, 1.25-6.71; $P=0.014$ ) (Table 3).
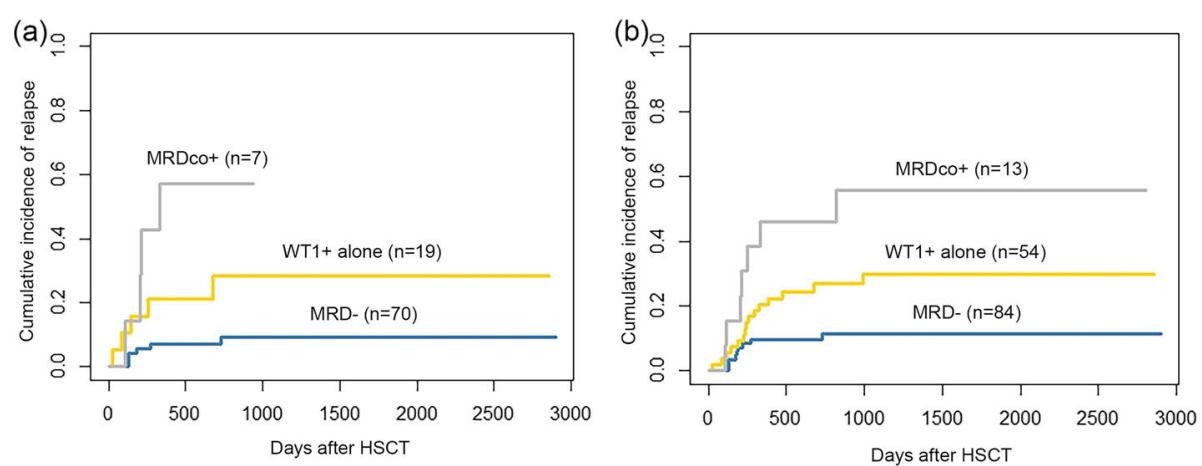

Fig. 3 The relapse of patients according to MRD status after allo-HSCT. a 2-year CIR in patients without any preemptive interventions ( $n=96)$ : WT1+ alone vs. MRD-: $28.4 \%$ vs. $9.2 \%, P=0.032 ;$ MRDco+ vs. MRD-: $57.1 \%$ vs. $9.2 \%, P<0.001 ;$ WT1+ alone vs. MRDco+: $28.4 \%$ vs. $57.1 \% ; P=0.168$. b 5 -year CIR in all patients $(n=151)$ : WT1+ alone vs. MRD-: $29.8 \%$ vs. $11.3 \%, P=0.013$; MRDco+ vs. MRD-: $55.8 \%$ vs. $11.3 \%, P<0.001$; WT1+ alone vs. MRDco+: $29.8 \%$ vs. $55.8 \%, P=0.060$ 
Table 2 Multivariate analyses for 2-year outcomes in patients without preemptive interventions $(n=96)$

\begin{tabular}{|c|c|c|}
\hline Variables & HR $(95 \% \mathrm{Cl})$ & $P$ value \\
\hline \multicolumn{3}{|l|}{ Relapse } \\
\hline \multicolumn{3}{|c|}{ Cytogenetics and molecular abnormalities } \\
\hline Intermediate & 1.00 & \\
\hline Unfavorable & $3.41(1.08-10.81)$ & $0.037^{*}$ \\
\hline \multicolumn{3}{|c|}{ Disease status before allo-HSCT } \\
\hline CR1 & 1.00 & \\
\hline CR2 & $2.03(0.39-10.43)$ & 0.398 \\
\hline NR & $114.16(13.86-940.41)$ & $<0.001^{*}$ \\
\hline \multicolumn{3}{|c|}{ MRD status after allo-HSCT } \\
\hline MRD- & 1.00 & \\
\hline WT1+ alone & $6.15(1.62-23.33)$ & $0.008^{*}$ \\
\hline \multirow[t]{2}{*}{ MRDco+ } & $14.70(3.63-59.58)$ & $<0.001^{*}$ \\
\hline & DFS & \\
\hline \multicolumn{3}{|c|}{ Cytogenetics and molecular abnormalities } \\
\hline Intermediate & 1.00 & \\
\hline Unfavorable & $2.88(1.15-7.20)$ & $0.024^{*}$ \\
\hline \multicolumn{3}{|c|}{ Disease status before allo-HSCT } \\
\hline CR1 & 1.00 & \\
\hline CR2 & $1.11(0.24-5.04)$ & 0.896 \\
\hline NR & $33.06(5.94-184.00)$ & $<0.001^{*}$ \\
\hline \multicolumn{3}{|c|}{ MRD status after allo-HSCT } \\
\hline MRD- & 1.00 & \\
\hline WT1+ alone & $2.97(1.05-8.46)$ & $0.041^{*}$ \\
\hline \multirow[t]{2}{*}{ MRDco+ } & $6.37(1.94-20.99)$ & $0.002^{*}$ \\
\hline & OS & \\
\hline \multicolumn{3}{|c|}{ Cytogenetics and molecular abnormalities } \\
\hline Intermediate & 1.00 & \\
\hline Unfavorable & $2.90(1.10-7.66)$ & $0.031^{*}$ \\
\hline \multicolumn{3}{|c|}{ Disease status before allo-HSCT } \\
\hline CR1 & 1.00 & \\
\hline CR2 & $0.47(0.06-3.66)$ & 0.472 \\
\hline NR & $14.97(2.95-76.02)$ & $0.001^{*}$ \\
\hline \multicolumn{3}{|c|}{ MRD status after allo-HSCT } \\
\hline MRD- & 1.00 & \\
\hline WT1+ alone & $1.62(0.49-5.42)$ & 0.433 \\
\hline MRDco+ & $7.13(2.11-24.07)$ & $0.002^{*}$ \\
\hline
\end{tabular}

CR Complete remission, NR No remission, MRD- MRD negativity, WT1+ alone WT1 positivity alone, MRDco+ Combined MRD positivity, DFS Disease-free survival, OS Overall survival, HR Hazard ratio, Cl Confidence interval

* means $P<0.05$

In total, 111 patients received haplo-RD in CR1. Among them, patients with WT1 positivity after alloHSCT had a significantly higher 5-year CIR (34.8\% vs. $4.0 \% ; P<0.001)$, a lower 5 -year DFS $(61.0 \%$ vs. $85.6 \%$; $P=0.005)$, and a lower 5 -year OS $(64.4 \%$ vs. $85.5 \% ; P=$
0.017) than those with WT1 negativity. Eighteen patients received HLA-identical sibling donor (ISD) transplantation or URD, six of them relapsed, and three relapsed patients $(50 \%)$ were WT1 positive. The other 12 patients did not relapse, and seven of them (58\%) were WT1 positive.

The CIR of WT1 positive patients was compared with that of WT1 negative patients at each monitoring time point $(1,2,3,4.5,6,9$, and 12 months posttransplantation). Patients with WT1 positivity at +1 month (5-year CIR: $30.8 \%$ vs. $20.1 \% ; P=0.277$ ),+3 months (5-year CIR: $16.7 \%$ vs. $21.2 \% ; P=0.834$ ),+4.5 months (5-year CIR: $14.6 \%$ vs.19.6\%; $P=0.646$ ), and + 12 months (5-year CIR: $16.7 \%$ vs. $2.1 \% ; P=0.067$ ) were not likely to relapse more than those with WT1 negativity. Nevertheless, patients with WT1 positivity at +2 months +6 months and +9 months relapsed more than those with WT1 negativity. Patients with WT1 positivity at +2 months had a significantly higher 5 -year CIR ( $40.0 \%$ vs. $20.2 \% ; P=0.019)$, a lower 5 -year DFS (46.7\% vs. $75.4 \% ; P<0.001)$, and a lower 5 -year OS $(41.5 \%$ vs. $78.8 \%$; $P<0.001)$ than those with WT1 negativity. Patients with WT1 positivity at +6 months had a significantly higher 5 -year CIR $(28.6 \%$ vs. $14.3 \%$; $P=$ $0.035)$ than those with WT1 negativity, but the DFS and OS showed no difference between these two groups. Patients with WT1 positivity at +9 months had a significantly higher 5 -year CIR (32.5\% vs. $3.9 \% ; P=0.002)$, a lower 5 -year DFS $(67.5 \%$ vs. $92.5 \% ; P=0.014)$, and a lower 5 -year OS $(70.3 \%$ vs. $94.3 \%$; $P=0.036)$ than those with WT1 negativity.

\section{Preemptive intervention after allo-HSCT}

Among the 67 patients who showed MRD positivity within 1 year after allo-HSCT (WT1+ alone: $n=54$; MRDco+: $n=13), 25$ received preemptive IFN- $\alpha$ treatment alone. Sixteen patients (64\%) achieved MRD negativity after IFN- $\alpha$ treatment, with a median of 43 (range, 10 to 542) days from IFN- $\alpha$ treatment to MRD turning negative. One patient relapsed after achieving MRD negativity. The CIR (5-year CIR: $8.3 \%$ vs. $11.3 \% ; P=$ 0.513 ) and DFS (5-year DFS: $91.7 \%$ vs. $80.8 \% ; P=0.208$ ) were comparable between patients achieving MRD negativity after IFN- $\alpha$ treatment and those without MRD after allo-HSCT, which were both better than those of patients who had MRD but did not undergo any preemptive therapies (Fig. 4). Among the 9 patients who showed persistent MRD after IFN- $\alpha$ treatment, 3 relapsed, and the median time from IFN- $\alpha$ treatment to relapse was 70 days (range, 9 to 125 ).

Sixteen patients received preemptive Chemo-DLI (WT1+ alone: $n=10$; MRDco+: $n=6$ ), including 10 patients receiving IFN- $\alpha$ treatment prior to Chemo-DLI (persistent MRD after IFN- $\alpha$ treatment: $n=8$; regained 


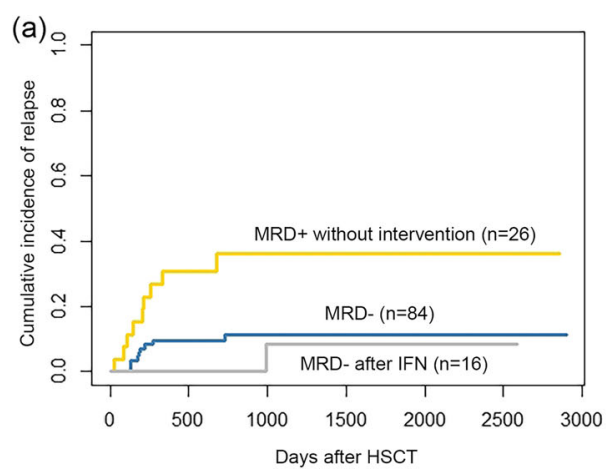

(b)

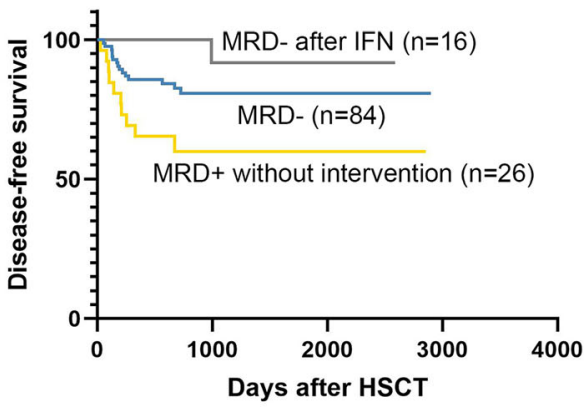

Fig. 4 The outcomes of patients according to preemptive IFN-a treatment. a 5-year CIR: MRD- after IFN vs. MRD-: 8.3\% vs. 11.3\%, $P=0.513$; MRDafter IFN vs. MRD+ without intervention: $8.3 \%$ vs. $36.2 \%, P=0.024$; MRD- vs. MRD+ without intervention: $11.3 \%$ vs. $36.2 \%, P=0.004$. b 5-year DFS: MRD- after IFN vs. MRD-: $91.7 \%$ vs. $80.8 \%, P=0.208$; MRD- after IFN vs. MRD+ without intervention: $91.7 \%$ vs. $59.9 \%, P=0.014 ;$ MRD- vs. MRD+ without intervention: $80.8 \%$ vs. $59.9 \%, P=0.019$

MRD positivity after achieving MRD negativity: $n=2$ ). Four (25\%) patients achieved MRD negativity after Chemo-DLI, with a median of 31.5 (range, 30 to 32) days from Chemo-DLI to MRD turning negative. Among the 4 patients achieving MRD negativity, 1 (25\%) showed DFS after Chemo-DLI, 1 (25\%) suffered from NRM, and 2 (50\%) experienced relapse. Among the 12 patients who showed persistent MRD after Chemo-DLI, 7 (58.3\%) experienced relapse.

\section{WT1 positivity over 1 year after Allo-HSCT}

In total, 56 patients who achieved DFS without preemptive intervention had data of WT1 expression over 1 year after allo-HSCT. In these patients, the median WT1 expression levels at $+18,+24,+30,+36,+48$, and +60 months were $0.280 \%(0.020-1.100 \%), 0.245 \%$ (0.041$0.860 \%), 0.300 \%$ (0.042-1.500\%), 0.260\% (0.085-1.200\%), $0.210 \%(0.055-0.370 \%)$, and $0.200 \%(0.015-1.300 \%)$, respectively (eFigure 3 in the Supplement). Four (4/56) patients were WT1 positive. Among them, two were transient WT1 positive, while the other two patients were persistent WT1 positive and had cGVHD.

In total, 31 patients relapsed after allo-HSCT. Among them, 25 patients relapsed within 1 year after allo-HSCT. Four patients received preemptive intervention within 1 year after allo-HSCT. Thus, only two relapsed patients had data of WT1 expression over 1 year after allo-HSCT and WT1 expression levels were both negative $(<0.8 \%)$ in these two patients.

Spearman correlation analysis showed that WT1 positivity was not associated with MFC positivity (Spearman's correlation coefficient: $-0.043, P=0.744$ ) over 1 year after allo-HSCT.

\section{Discussion}

Our study observed that the relapse rate of pediatric AML patients who had WT1 expression $\geq 0.8 \%$ within 1 year after transplantation was significantly higher than that of patients with WT1 expression $<0.8 \%$. Moreover, preemptive IFN- $\alpha$ treatment could help to clear MRD, decrease the risk of relapse, and improve DFS. To the best of our knowledge, our study shows the first relapse prediction result of WT1 sequential monitoring after allo-HSCT in a disease-specific population of children with AML, and these results provide an opportunity for exploring the up-to-date undefined predictive role of WT1 in these patients.

A previous study monitored WT1 expression at 2 time points after allo-HSCT $(+42 \mathrm{~d}$ and $+100 \mathrm{~d})$ and showed that WT1 could not predict relapse in pediatric AML [16]. Using only 2 time points to monitor WT1 may miss data of other important time points. Relapsed patients with normal WT1 expression at 2 time points measured may actually have elevated WT1 at other time points without detection. We performed WT1 monitoring at $1,2,3,4.5,6,9$, and 12 months posttransplantation, which was sequential monitoring and could lower the frequency of false negativity. Compared to the previous study [16], we monitored WT1 expression after 3 months post-transplantation additionally. Our data showed that WT1 monitoring at +6 months +9 months could also predict relapse, which suggested that monitoring WT1 expression after 3 months post-transplantation was also reasonable.

Based on ROC analysis, we defined WT1 positivity as WT $1 \geq 0.8 \%$, while WT $1 \geq 0.6 \%$ was considered WT1 positivity in our previous studies enrolling adult patients $[11,12]$. A previous study reported that children had a higher WT1 expression level than adults under physiological conditions [15]. Because WT1 was not a leukemia-specific molecular marker, a relatively higher cut-off value could spare pediatric AML patients who had slightly elevated WT1 levels but were actually in molecular remission from further interventions. 
Table 3 Multivariate analyses for 5-year outcomes in the total patients $(n=151)$

\begin{tabular}{|c|c|c|}
\hline Variables & $\mathrm{HR}(95 \% \mathrm{Cl})$ & $P$ value \\
\hline \multicolumn{3}{|l|}{ Relapse } \\
\hline \multicolumn{3}{|c|}{ Cytogenetics and molecular abnormalities } \\
\hline Intermediate & 1.00 & \\
\hline Unfavorable & $2.23(1.05-4.75)$ & $0.037^{*}$ \\
\hline \multicolumn{3}{|c|}{ Disease status before allo-HSCT } \\
\hline CR1 & 1.00 & \\
\hline CR2 & $1.93(0.55-6.81)$ & 0.306 \\
\hline NR & $10.74(3.25-35.47)$ & $<0.001^{*}$ \\
\hline \multicolumn{3}{|l|}{ Donor type } \\
\hline ISD & 1.00 & \\
\hline URD & $0.32(0.04-2.82)$ & 0.302 \\
\hline Haplo-RD & $0.33(0.12-0.90)$ & $0.030^{*}$ \\
\hline UCB & $1.85(0.35-9.86)$ & 0.472 \\
\hline \multicolumn{3}{|c|}{ MRD status after allo-HSCT } \\
\hline MRD- & 1.00 & \\
\hline WT1+ alone & $2.89(1.25-6.71)$ & $0.014^{*}$ \\
\hline MRDco+ & $6.10(2.09-17.78)$ & $0.001^{*}$ \\
\hline \multicolumn{3}{|c|}{ DFS } \\
\hline \multicolumn{3}{|c|}{ Cytogenetics and molecular abnormalities } \\
\hline Intermediate & 1.00 & \\
\hline Unfavorable & $2.49(1.32-4.72)$ & $0.005^{*}$ \\
\hline \multicolumn{3}{|c|}{ Disease status before allo-HSCT } \\
\hline CR1 & 1.00 & \\
\hline CR2 & $1.48(0.51-4.27)$ & 0.471 \\
\hline NR & $6.23(2.06-18.81)$ & $0.001^{*}$ \\
\hline \multicolumn{3}{|c|}{ MRD status after allo-HSCT } \\
\hline MRD- & 1.00 & \\
\hline WT1+ alone & $1.79(0.88-3.65)$ & 0.110 \\
\hline MRDco+ & $4.75(2.00-11.29)$ & $<0.001^{*}$ \\
\hline \multicolumn{3}{|c|}{ OS } \\
\hline \multicolumn{3}{|c|}{ Cytogenetics and molecular abnormalities } \\
\hline Intermediate & 1.00 & \\
\hline Unfavorable & $2.42(1.24-4.73)$ & $0.010^{*}$ \\
\hline \multicolumn{3}{|c|}{ MRD status after allo-HSCT } \\
\hline MRD- & 1.00 & \\
\hline WT1+ alone & $1.59(0.74-3.38)$ & 0.232 \\
\hline MRDco+ & $5.62(2.34-13.52)$ & $<0.001^{*}$ \\
\hline \multicolumn{3}{|c|}{$\begin{array}{l}\text { CR Complete remission, NR No remission, ISD HLA-identical sibling donor, URD } \\
\text { HLA-unrelated donor, Haplo-RD HLA-haploidentical related donor, UCB } \\
\text { Umbilical cord blood, MRD- MRD negativity, WT1+ alone WT1 positivity alone, } \\
\text { MRDco+ Combined MRD positivity, DFS Disease-free survival, OS Overall } \\
\text { survival, HR Hazard ratio, CI Confidence interval } \\
{ }^{*} \text { means } P<0.05\end{array}$} \\
\hline
\end{tabular}

MRD could be monitored by MFC in AML, and MRD positivity was recommended to be defined as MFC > $0.1 \%$ [5]. Therefore, MRD detected by MFC might not be sensitive enough to predict relapse and direct preemptive intervention. In this study, we found that WT1 was significantly associated with MFC, and some patients showed WT1 positivity prior to MFC positivity with a median interval of 95 days. These results suggested that WT1 could reflect the residual leukemia cells in AML children and could be used as an MRD marker in these patients. Nevertheless, the association between WT1 positivity and MFC positivity was not so strong in the study. A large scale, multicenter study is needed to explore the relationship between WT1 and MFC in the future. In addition, we observed that patients with WT1+ alone had a trend of a lower CIR than those with MRDco+ (i.e., WT1+ and MFC+), which suggested that there might be a lower leukemia burden for patients with WT1+ alone. Because immunotherapy should preferably be started in patients with a relatively low leukemia burden [31], WT1-directed immunotherapy may help to clear MRD more promptly and effectively.

In order to remove the influence of population heterogeneity, we restricted prognostic analysis to patients who received haplo-RD in CR1. Results showed that patients with WT1 positivity had worse prognosis than those with WT1 negativity. This result was in consistent with that of the whole cohort. In patients who received ISD or URD, the ratio of WT1 positivity (50\% vs. $58 \%$ ) was not different between relapse and non-relapse groups. But it was hard to draw a conclusion about the prognostic role of WT1 expression in patients receiving ISD or URD because of the limited patients included in the study. Thus, in the future, a large scale and multicenter study is needed to address this issue.

Theoretically, the kinetics of MRD could predict prognosis. Since the influence of preemptive intervention should be removed, WT1 positive patients without preemptive intervention were used for analyses. Nine patients relapsed, and eight of them were WT1 positive once before relapse, while only one patient showed WT1 positive more than once before relapse. Thus, since there was only one relapsed patient with WT1 positivity more than once, we failed to explore the prognostic role of the kinetics of WT1 expression. A prospective, large scale and multicenter study is needed to explore the prognostic value of the kinetics of WT1 expression in the future.

WT1 was not a gold standard for starting preemptive intervention as relapse prediction of WT1 in pediatric AML after allo-HSCT was unclear. Doctors and patients started preemptive intervention depending on their own intention. However, many studies showed that WT1 could guide preemptive intervention in adult AML after allo-HSCT [11, 12, 23-30]. Therefore, we wanted to see 
whether pediatric AML patients could benefit from WT1-directed preemptive intervention. In patients with WT1 positivity determined by ROC analysis, we analyzed whether patients benefited from WT1-directed immunotherapy by comparing the outcomes of patients receiving preemptive intervention with those without it. We separated the whole patients into four groups (MRD negativity, MRD positivity without preemptive intervention, MRD positivity treated with IFN- $\alpha$, MRD positivity treated with Chemo-DLI) to perform analysis, which may help to lower the impact of population heterogeneity on patients' outcomes.

IFN- $\alpha$ treatment could clear MRD effectively [23, 25-28]. Our study found that $64 \%$ of pediatric AML patients achieved MRD negativity after preemptive IFN- $\alpha$ monotherapy. In addition, the CIR of patients who achieved WT1 negativity after IFN- $\alpha$ treatment was comparable to that of those without MRD after allo-HSCT, which was lower than that of WT1-positive patients without any preemptive interventions. These results were in accordance with those of our previous studies [23, 25-28]. It is suggested that IFN- $\alpha$ treatment could partially overcome the poor prognostic significance of increased WT1 expression after allo-HSCT, which further confirmed that WT1 could be used as an MRD marker in AML children. Several patients with persistent MRD after IFN- $\alpha$ treatment did not relapse. WT1 was not a leukemia-specific molecular marker. Thus, it was unavoidable that some patients may receive IFN- $\alpha$ treatment based on elevated WT1 which was not relative to leukemia in fact. However, IFN- $\alpha$ could reduce relapse in patients with WT1 positivity, which suggested that most patients could benefit from WT1-directed IFN- $\alpha$ treatment. In addition, IFN- $\alpha$ was safe and brought a very low risk of severe side effects. Few pediatric AML patients got MRD negativity nearly 2 weeks after IFN- $\alpha$ treatment. According to previous research [31], the earlier patients with positive MRD started immunotherapy, the better results they would get because of low leukemia burden at the time of preemptive intervention. Despite this, the median interval from IFN- $\alpha$ to MRD turning negativity was 43 days. Maybe there was false positivity of WT1, but this was the inevitable defect of any MRD detection method. Actually, our study showed that WT1 had relatively high sensitivity and specificity to predict relapse in pediatric AML patients after allo-HSCT.

It seemed that the efficacy of DLI was not as good as that observed in our previous studies $[24,29,30]$. This may be because most patients $(62.5 \%)$ showed persistent MRD or regained MRD positivity after achieving negativity after prior IFN- $\alpha$ treatment. That is, Chemo-DLI was used as salvage therapy in these patients. Graftversus-leukemia (GVL) is the critical mechanism of clearing MRD for both IFN- $\alpha$ and DLI, so an unsatisfactory response to IFN- $\alpha$ treatment suggests that residual leukemia cells may have immune escape and may not be sensitive to other immunotherapies (e.g., DLI) after alloHSCT. Similarly, our previous study observed that the efficacy of salvage Chemo-DLI for IFN- $\alpha$ treatment was not sufficient [26]. Thus, how to further clear the MRD of these patients should be further studied. However, due to the small number of DLI cases, it would be premature to derive conclusions regarding the efficacy of Chemo-DLI in children with AML.

The prognostic role of WT1 expression and preemptive intervention directed by WT1 expression within 1 year after allo-HSCT had been discussed above. Although we also monitored WT1 expression over 1 year after allo-HSCT, the monitoring intervals were at least 6 months, which may not unveil the change of WT1 expression completely. Since patients included in the relapse group was not enough $(n=2)$, we failed to compare the WT1 expression levels between relapse and non-relapse groups. A prospective, large scale and multicenter study is needed to address this issue in the future.

Chimerism was not detected regularly at our center. The sensitivity of chimerism to predict relapse was lower than MRD, and mixed chimerism was not equal to relapse [32].

Our study had the following limitations. First, the study was a retrospective study. In addition, the number of patients included in the study was not large enough, particularly the number of patients receiving preemptive interventions. Thus, we could not further compare the efficacy between IFN- $\alpha$ treatment and Chemo-DLI in these patients. Future randomized, prospective trials may further compare the efficacy of these interventions. In addition, no patients received hypomethylating agents in the present study. These agents are worth further study, particularly for those who showed unsatisfactory responses to immunotherapies. Last, because the scale of our study was not much large, we were unable to perform test set analysis.

\section{Conclusions}

In conclusion, WT1 sequential monitoring was able to predict relapse in pediatric AML patients after alloHSCT. In addition, WT1-directed immunotherapy may have the potential to prevent relapse and improve survival in AML children after allo-HSCT.

\footnotetext{
Abbreviations

AML: Acute myeloid leukemia; Ara-C: Cytarabine; BM: Bone marrow; CsA: Cyclosporine A; CR: Complete remission; Cl: Confidence interval; CIR: Cumulative incidence of relapse; Chemo-DLI: Chemotherapy plus donor lymphocyte infusion; DFS: Disease-free survival; haplo-RD: HLA-haploidentical related donor; IFN-a: Interferon-a; LAIPs: Leukemia-associated aberrant immune phenotypes; MRD: Minimal residual disease; MFC: Multiparameter flow cytometry; MMF: Mycophenolate mofetil; MTX: Methotrexate; MP: Methylprednisolone; MRDco+: Combined MRD positivity; NRM: Nonrelapse mortality; OS: Overall survival; PCR: Polymerase chain reaction; URD: HLA-unrelated donor; UCB: Umbilical cord blood; WT1: Wilms' tumor gene 1; WT1+ alone: WT1 positivity alone
} 


\section{Supplementary Information}

The online version contains supplementary material available at https://doi. org/10.1186/s12885-021-08022-0.

Additional file 1: eMethods. eTable 1. The sensitivity and specificity of different cut-off value for WT1. eFigure 1. ROC curve of WT1 expression level and the relapse rate $(n=96)$. eFigure $\mathbf{2}$. The outcomes of the total patients according to WT1 after allo-HSCT $(n=151)$. eFigure $\mathbf{3}$. WT1 expression at different points over 1 year after allo-HSCT in patients maintaining CR without interventions $(n=56)$.

\section{Acknowledgements}

Not applicable.

\section{Authors' contributions}

X.-D.M. and X.-S.Z. designed the study; Y.-F.C., X.-H.Z., L.-P.X., Y.W., C.-H.Y., Y.H.C., H.C., W.H., F.-R.W., J.-Z.W., Y.-Z.Q., K.-Y.L., and X.-J.H. collected and assembled the data; D.-X.D., J.-J.W., X.-D.M., and X.-S.Z. analyzed and interpreted the data; D.-X.D., J.-J.W., X.-D.M., and X.-S.Z. wrote the manuscript; and all authors read and approved the final manuscript.

\section{Funding}

This work was supported by grants from CAMS Innovation Fund for Medical Sciences (CIFMS) (grant no. 2019-I2M-5-034), the National Key Research and Development Program of China (No. 2017YFA0104500), Innovative Research Groups of the National Natural Science Foundation of China (No. 81621001), the Key Program of the National Natural Science Foundation of China (No.81930004), and the Capital's Funds for Health Improvement and Research (grant no. 2018-4-4089).

\section{Availability of data and materials}

The datasets used and analyzed during the current study are available from the corresponding author on reasonable request.

\section{Declarations}

\section{Ethics approval and consent to participate}

This study followed the principles of the Helsinki Declaration and was approved by the ethical committee of Peking University People's Hospital. All patient guardians had signed informed consent.

\section{Consent for publication}

Not applicable.

\section{Competing interests}

The authors declare that they have no competing interests.

\section{Author details}

${ }^{1}$ Peking University People's Hospital, Peking University Institute of Hematology, National Clinical Research Center for Hematologic Disease, Research Unit of Key Technique for Diagnosis and Treatments of Hematologic Malignancies, Chinese Academy of Medical Sciences, 2019RU029, Beijing Key Laboratory of Hematopoietic Stem Cell Transplantation, Beijing, China. ${ }^{2}$ Department of Hematology, Peking University Shenzhen Hospital, Shenzhen, China. ${ }^{3}$ Peking-Tsinghua Center for Life Sciences, Beijing, China. ${ }^{4}$ Collaborative Innovation Center of Hematology, Peking University, Beijing, China.

Received: 24 December 2020 Accepted: 8 March 2021

Published online: 19 March 2021

\section{References}

1. Hasle H. A critical review of which children with acute myeloid leukaemia need stem cell procedures. Br J Haematol. 2014;166(1):23-33. https://doi. org/10.1111/bjh.12900

2. Xu L, Chen $H$, Chen J, Han M, Huang H, Lai Y, Liu D, Liu Q, Liu T, Jiang M, Ren $H$, Song Y, Sun Z, Wang J, Wu D, Zhou D, Zou P, Liu K, Huang X. The consensus on indications, conditioning regimen, and donor selection of allogeneic hematopoietic cell transplantation for hematological diseases in
China-recommendations from the Chinese Society of Hematology. J Hematol Oncol. 2018;11(1):33. https://doi.org/10.1186/s13045-018-0564-x.

3. Wang $Y$, Chen $H$, Chen J, Han M, Hu J, Jiong H, et al. The consensus on the monitoring, treatment, and prevention of leukemia relapse after allogeneic hematopoietic stem cell transplantation in China. Cancer Lett. 2018;438:6375. https://doi.org/10.1016/j.canlet.2018.08.030.

4. Ravandi F, Walter RB, Freeman SD. Evaluating measurable residual disease in acute myeloid leukemia. Blood Adv. 2018;2(11):1356-66. https://doi.org/1 $0.1182 /$ bloodadvances.2018016378.

5. Schuurhuis GJ, Heuser M, Freeman S, Bene MC, Buccisano F, Cloos J, et al. Minimal/measurable residual disease in AML: a consensus document from the European LeukemiaNet MRD Working Party. Blood. 2018;131(12):127591. https://doi.org/10.1182/blood-2017-09-801498.

6. Paietta E. Minimal residual disease in AML: why has it lagged behind pediatric ALL? Clin Lymphoma Myeloma Leuk. 2015;15(Suppl):S2-6. https:// doi.org/10.1016/j.clml.2015.02.009.

7. Rizzari C, Cazzaniga G, Coliva T, De Angelis C, Conter V. Predictive factors of relapse and survival in childhood acute myeloid leukemia: role of minimal residual disease. Expert Rev Anticancer Ther. 2011;11(9):1391-401. https:// doi.org/10.1586/era.11.37.

8. Creutzig U, van den Heuvel-Eibrink MM, Gibson B, Dworzak MN, Adachi S, de Bont E, Harbott J, Hasle H, Johnston D, Kinoshita A, Lehrnbecher T, Leverger G, Mejstrikova E, Meshinchi S, Pession A, Raimondi SC, Sung L, Stary J, Zwaan CM, Kaspers GJ, Reinhardt D, AML Committee of the International BFM Study Group. Diagnosis and management of acute myeloid leukemia in children and adolescents: recommendations from an international expert panel. Blood. 2012;120(16):3187-205. https://doi.org/1 0.1182/blood-2012-03-362608.

9. Shimada A, Taki T, Koga D, Tabuchi K, Tawa A, Hanada R, Tsuchida M, Horibe K, Tsukimoto I, Adachi S, Kojima S, Hayashi Y. High WT1 mRNA expression after induction chemotherapy and FLT3-ITD have prognostic impact in pediatric acute myeloid leukemia: a study of the Japanese Childhood AML Cooperative Study Group. Int J Hematol. 2012;96(4):469-76. https://doi.org/10.1007/s12185-012-1163-1.

10. Zhang R, Yang JY, Sun HQ, Jia H, Liao J, Shi YJ, Li G. Comparison of minimal residual disease (MRD) monitoring by $\mathrm{WT1}$ quantification between childhood acute myeloid leukemia and acute lymphoblastic leukemia. Eur Rev Med Pharmacol Sci. 2015;19(14):2679-88.

11. Zhao XS, Jin S, Zhu HH, Xu LP, Liu DH, Chen H, Liu KY, Huang XJ. Wilms' tumor gene 1 expression: an independent acute leukemia prognostic indicator following allogeneic hematopoietic SCT. Bone Marrow Transplant. 2012;47(4):499-507. https://doi.org/10.1038/bmt.2011.121.

12. Zhao XS, Yan CH, Liu DH, Xu LP, Liu YR, Liu KY, Qin YZ, Wang Y, Huang XJ. Combined use of WT1 and flow cytometry monitoring can promote sensitivity of predicting relapse after allogeneic HSCT without affecting specificity. Ann Hematol. 2013;92(8):1111-9. https://doi.org/10.1007/s00277013-1733-1.

13. Pozzi S, Geroldi S, Tedone E, Luchetti S, Grasso R, Colombo N, di Grazia C, Lamparelli T, Gualandi F, Ibatici A, Bregante S, van Lint MT, Raiola AM, Dominietto A, Varaldo R, Signori A, Bacigalupo A. Leukaemia relapse after allogeneic transplants for acute myeloid leukaemia: predictive role of WT1 expression. Br J Haematol. 2013;160(4):503-9. https://doi.org/10.1111/bjh.121 81.

14. Dulery R, Nibourel O, Gauthier J, Elsermans V, Behal H, Coiteux V, et al. Impact of Wilms' tumor 1 expression on outcome of patients undergoing allogeneic stem cell transplantation for AML. Bone Marrow Transplant. 2017; 52(4):539-43. https://doi.org/10.1038/bmt.2016.318.

15. Lovvik Juul-Dam K, Guldborg Nyvold C, Valerhaugen H, Zeller B, Lausen B, Hasle $\mathrm{H}$, et al. Measurable residual disease monitoring using Wilms tumor gene 1 expression in childhood acute myeloid leukemia based on childspecific reference values. Pediatr Blood Cancer. 2019;66(6):e27671. https:// doi.org/10.1002/pbc.27671.

16. Jacobsohn DA, Loken MR, Fei M, Adams A, Brodersen LE, Logan BR, Ahn KW, Shaw BE, Kletzel M, Olszewski M, Khan S, Meshinchi S, Keating A, Harris A, Teira P, Duerst RE, Margossian SP, Martin PL, Petrovic A, Dvorak CC, Nemecek ER, Boyer MW, Chen AR, Davis JH, Shenoy S, Savasan S, Hudspeth MP, Adams RH, Lewis VA, Kheradpour A, Kasow KA, Gillio AP, Haight AE, Bhatia M, Bambach BJ, Haines HL, Quigg TC, Greiner RJ, Talano JAM, Delgado DC, Cheerva A, Gowda M, Ahuja S, Ozkaynak M, Mitchell D, Schultz KR, Fry TJ, Loeb DM, Pulsipher MA. Outcomes of measurable residual disease in pediatric acute myeloid leukemia before and after hematopoietic 
stem cell transplant: validation of difference from normal flow cytometry with Chimerism studies and Wilms tumor 1 gene expression. Biol Blood Marrow Transplant. 2018;24(10):2040-6. https://doi.org/10.1016/j.bbmt.2018. 06.010.

17. Xiao-Jun H, Lan-Ping X, Kai-Yan L, Dai-Hong L, Yu W, Huan C, Yu-Hong C, Wei H, Jing-Zhi W, Yao C, Xiao-Hui Z, Hong-Xia S, Feng-Rong W, Fei-Fei T. Partially matched related donor transplantation can achieve outcomes comparable with unrelated donor transplantation for patients with hematologic malignancies. Clin Cancer Res. 2009;15(14):4777-83. https://doi. org/10.1158/1078-0432.CCR-09-0691.

18. Wang Y, Liu QF, Xu LP, Liu KY, Zhang XH, Ma X, Fan ZP, Wu DP, Huang XJ. Haploidentical vs identical-sibling transplant for AML in remission: a multicenter, prospective study. Blood. 2015;125(25):3956-62. https://doi. org/10.1182/blood-2015-02-627786.

19. Mo XD, Zhao XY, Liu DH, Chen YH, Xu LP, Zhang XH, Chen H, Han W, Wang Y, Wang FR, Wang JZ, Liu KY, Huang XJ. Umbilical cord blood transplantation and unmanipulated haploidentical hematopoietic SCT for pediatric hematologic malignances. Bone Marrow Transplant. 2014;49(8): 1070-5. https://doi.org/10.1038/bmt.2014.109.

20. Mo X-D, Zhang Y-Y, Zhang X-H, Xu L-P, Wang Y, Yan C-H, Chen H, Chen $Y-H$, Chang $Y-J$, Liu K-Y, Huang $X-J$. The role of collateral related donors in haploidentical hematopoietic stem cell transplantation[J]. Sci Bull. 2018,63(20):1376-82. https://doi.org/10.1016/j.scib.2018.08.008.

21. Huang XJ, Liu DH, Liu KY, Xu LP, Chen H, Han W, Chen YH, Zhang XH, Lu DP. Treatment of acute leukemia with unmanipulated HLA-mismatched/ haploidentical blood and bone marrow transplantation. Biol Blood Marrow Transplant. 2009;15(2):257-65. https://doi.org/10.1016/j.bbmt.2008.11.025.

22. Chang YJ, Wang Y, Liu YR, Xu LP, Zhang XH, Chen H, Chen YH, Wang FR, Han W, Sun YQ, Yan CH, Tang FF, Mo XD, Liu KY, Huang XJ. Haploidentical allograft is superior to matched sibling donor allograft in eradicating pretransplantation minimal residual disease of AML patients as determined by multiparameter flow cytometry: a retrospective and prospective analysis. J Hematol Oncol. 2017;10(1):134. https://doi.org/10.1186/s13045-017-0502-3.

23. Mo XD, Zhang XH, Xu LP, Wang Y, Yan CH, Chen H, Chen YH, Han W, Wang FR, Wang JZ, Liu KY, Huang XJ. Interferon-alpha: a potentially effective treatment for minimal residual disease in acute leukemia/Myelodysplastic syndrome after allogeneic hematopoietic stem cell transplantation. Biol Blood Marrow Transplant. 2015;21(11):1939-47. https://doi.org/10.1016/j. bbmt.2015.06.014.

24. Yan CH, Liu DH, Liu KY, Xu LP, Liu YR, Chen H, Han W, Wang Y, Qin YZ, Huang XJ. Risk stratification-directed donor lymphocyte infusion could reduce relapse of standard-risk acute leukemia patients after allogeneic hematopoietic stem cell transplantation. Blood. 2012;119(14):3256-62. https://doi.org/10.1182/blood-2011-09-380386.

25. Mo XD, Zhang XH, Xu LP, Wang Y, Yan CH, Chen H, Chen YH, Han W, Wang FR, Wang JZ, Liu KY, Huang XJ. IFN-alpha is effective for treatment of minimal residual disease in patients with acute leukemia after allogeneic hematopoietic stem cell transplantation: results of a registry study. Biol Blood Marrow Transplant. 2017;23(8):1303-10. https://doi.org/10.1016/j. bbmt.2017.04.023.

26. Mo XD, Wang $Y$, Zhang $X H$, Xu LP, Yan $C H$, Chen $H$, Chen YH, Qin YZ, Liu $K Y$, Huang $X J$. Interferon-alpha is effective for treatment of minimal residual disease in patients with $t(8 ; 21)$ acute myeloid leukemia after allogeneic hematopoietic stem cell transplantation: results of a prospective registry study. Oncologist. 2018;23(11):1349-57. https://doi.org/10.1634/ theoncologist.2017-0692

27. Mo X, Zhang X, Xu L, Wang Y, Yan C, Chen H, Chen Y, Han W, Wang F, Wang J, Liu K, Huang X. Minimal residual disease-directed immunotherapy for high-risk myelodysplastic syndrome after allogeneic hematopoietic stem cell transplantation. Front Med. 2019;13(3):354-64. https://doi.org/10.1007/ s11684-018-0665-5.

28. Mo X, Zhang X, Xu L, Wang Y, Yan C, Chen H, Chen Y, Han W, Wang F, Wang J, Liu K, Huang $X$. Interferon-alpha salvage treatment is effective for patients with acute leukemia/myelodysplastic syndrome with unsatisfactory response to minimal residual disease-directed donor lymphocyte infusion after allogeneic hematopoietic stem cell transplantation. Front Med. 2019; 13(2):238-49. https://doi.org/10.1007/s11684-017-0599-3.

29. Mo XD, Zhang XH, Xu LP, Wang Y, Yan CH, Chen H, Chen YH, Han W, Wang FR, Wang JZ, Liu KY, Huang XJ. Salvage chemotherapy followed by granulocyte colony-stimulating factor-primed donor leukocyte infusion with graft-vs.-host disease control for minimal residual disease in acute leukemia/ myelodysplastic syndrome after allogeneic hematopoietic stem cell transplantation: prognostic factors and clinical outcomes. Eur J Haematol. 2016;96(3):297-308. https://doi.org/10.1111/ejh.12591.

30. Mo XD, Zhang XH, Xu LP, Wang Y, Yan CH, Chen H, Chen YH, Han W, Wang FR, Wang JZ, Liu KY, Huang XJ. Comparison of outcomes after donor lymphocyte infusion with or without prior chemotherapy for minimal residual disease in acute leukemia/myelodysplastic syndrome after allogeneic hematopoietic stem cell transplantation. Ann Hematol. 2017; 96(5):829-38. https://doi.org/10.1007/s00277-017-2960-7.

31. Anguille $S$, Lion E, Willemen $Y$, Van Tendeloo VF, Berneman ZN, Smits EL. Interferon-alpha in acute myeloid leukemia: an old drug revisited. Leukemia. 2011;25(5):739-48. https://doi.org/10.1038/leu.2010.324.

32. Tsirigotis P, Byrne M, Schmid C, Baron F, Ciceri F, Esteve J, Gorin NC, Giebel S, Mohty M, Savani BN, Nagler A. Relapse of AML after hematopoietic stem cell transplantation: methods of monitoring and preventive strategies. A review from the ALWP of the EBMT. Bone Marrow Transplant. 2016;51(11): 1431-8. https://doi.org/10.1038/bmt.2016.167.

\section{Publisher's Note}

Springer Nature remains neutral with regard to jurisdictional claims in published maps and institutional affiliations.

\section{Ready to submit your research? Choose BMC and benefit from:}

- fast, convenient online submission

- thorough peer review by experienced researchers in your field

- rapid publication on acceptance

- support for research data, including large and complex data types

- gold Open Access which fosters wider collaboration and increased citations

- maximum visibility for your research: over $100 \mathrm{M}$ website views per year

At BMC, research is always in progress.

Learn more biomedcentral.com/submissions 